\title{
MATHEMATICAL MODELING OF TORSIONAL SURFACE WAVE PROPAGATION IN A NON-HOMOGENEOUS TRANSVERSE ISOTROPIC ELASTIC SOLID SEMI-INFINITE MEDIUM UNDER A LAYER
}

\author{
M. SETHI* \\ Department of Applied Sciences \\ Govt. Polytechnic College for Girls \\ Jalandhar, INDIA \\ E-mails: munish_sethi26@yahoo.co.in; munishsethi76@gmail.com
}

A. SHARMA and A. VASISHTH

Department of Applied Sciences

Chandigarh Engg. College

Landran, Mohali, INDIA

\begin{abstract}
The present paper deals with the mathematical modeling of the propagation of torsional surface waves in a non-homogeneous transverse isotropic elastic half-space under a rigid layer. Both rigidities and density of the half-space are assumed to vary inversely linearly with depth. Separation of variable method has been used to get the analytical solutions for the dispersion equation of the torsional surface waves. Also, the effects of nonhomogeneities on the phase velocity of torsional surface waves have been shown graphically. Also, dispersion equations have been derived for some particular cases, which are in complete agreement with some classical results.
\end{abstract}

Key words: non-homogeneous media, torsional surface waves, rigidity.

\section{Introduction}

The formulations and solutions of many problems of linear wave-propagation for homogeneous media are available in the literature of continuum mechanics of solids. In recent years, however, sufficient interest has arisen in the problem connected with bodies whose mechanical properties are functions of space, i.e., non-homogeneous bodies. This interest is mainly due to the advent of solid rocket propellants, polymeric materials and growing demand of engineering and industrial applications. Studies of wave propagation in the Earth stratum under loads have usually assumed that the Earth behaves to a first approximation as an ideal elastic or visco-elastic material. The stratura may be of finite depth or it may be so deep compared to the size of the loaded area that it can be regarded as a half-space. In either case the complete solutions to elastic or viscoelastic problems are known when material parameters are treated independent of position.

The formation of Earth strate in nature tends, however, to result in depth variations of these parameters and this may be due principally, either to stratification of different materials or to the effect of superincumbent pressure. The case of homogeneous viscoelastic half-space has been treated extensively in the literature. By comparison little has been done on the non-homogenous viscoelastic half-space when the material characteristics vary continuously with depth. This fact is likely to be true when the effects of overburden pressure predominate. Furthermore, these studies have been restricted to particular variations which have allowed the resulting simplification to be exploited.

\footnotetext{
${ }^{*}$ To whom correspondence should be addressed
} 
Earth is a spherical body having finite dimension and the elastic waves generated must receive the effect of the boundaries. Naturally, this phenomenon leads to the investigation of boundary waves or surface waves, i.e., the waves, which are confined to some surface during their propagation. Although much literature is available on the propagation of surface waves, i.e., Rayleigh waves, Love waves and Stoneley waves etc., in a non-homogeneous elastic media (monographs of Stoneley [1], Bullen [2], Ewing et al. [3], Hunters [4] and Jeffreys [5]), but very little literature is available on the propagation of one another type of surface wave called torsional surface wave whose amplitudes decay exponentially with distance from the free surface and are horizontally polarized but give a twist to the medium when it propagates. These waves often propagate during earthquakes and become responsible to some extent for the destruction on the earth crustal layer. Therefore seismologists have started taking a keen interest on the propagation of torsional surface waves in different geo-media.

Sezawa [6] studied the dispersion of elastic waves propagated on curved surfaces. The transmission of elastic waves through a stratified solid medium was studied by Thomson [7]. Haskell [8] studied the dispersion of surface waves in multilayered media. Biot [9] studied the influence of gravity on Rayleigh waves, assuming the force of gravity to create a type of initial stress of hydrostatic nature and the medium to be incompressible.

Propagation of Love waves in a non-homogeneous stratum of finite depth sandwiched between two semi infinite isotropic media has been studied earlier by Sinha [10]. Wave propagation in a thin two-layered laminated medium with couple initial stress was studied by Roy [11]. The idea of introducing a continuous reinforcement at every point of an elastic solid was given by Belfield et al. [12]. Datta [13] studied the effect of gravity on Rayleigh wave propagation in a homogeneous, isotropic elastic solid medium. Effects of irregularities on the propagation of guided SH waves were studied by Chattopadhyay et al. [14].

Goda [15] studied the effect of non-homogeneity and anisotropy on Stoneley waves. Gupta et al. [16] investigated the influence of linearly varying density and rigidity on torsional surface waves in an inhomogeneous crustal layer.

Some of the recent notable works on propagation of torsional waves in various media with different geometries were published by Georgiadis et al. [17], Dey and Sarkar [18], Selim [19], Ozturk and Akbbarov [20] and Gupta et al. [21]. Torsional wave propagation in a thin rectangular domain using an asymptotic approach was studied by Davini et al. [22] whereas Dey et al. [23] investigated the torsional surface wave in an elastic layer with void pores.

Recently, Sethi and Gupta [24, 25, 26] investigated the SH waves, surface waves in elastic and visco-elastic media.

In the present problem, we have considered the propagation of torsional surface waves in a nonhomogeneous transverse isotropic elastic half-space under a rigid layer. The expression for displacement and dispersion equation is found in the closed form. The dispersion curves are depicted by means of graphs for different values of non-homogeneity parameters. The influence of non-homogeneity parameters on the dimensionless phase velocity has been studied.

Here, the non-homogeneity in the half-space can be taken as

$$
\begin{aligned}
& \mu_{L}=\frac{\mu_{0}}{1+a z}, \\
& \mu_{T}=\frac{\mu_{0}}{1+b z}, \\
& \rho=\frac{\rho_{0}}{1+m z} \quad \text { where } \quad a>0, \quad b>0, \quad m>0
\end{aligned}
$$

where $a, b$ and $m$ are constants and have the dimensions that are inverse of length. 


\section{Formulation of problem and solution}

We consider a transversely isotropic elastic solid semi-infinite medium with inversely linearly varying rigidity and density with depth under a rigid layer. For the study of torsional waves, a cylindrical coordinate system is used as shown in Fig.1.

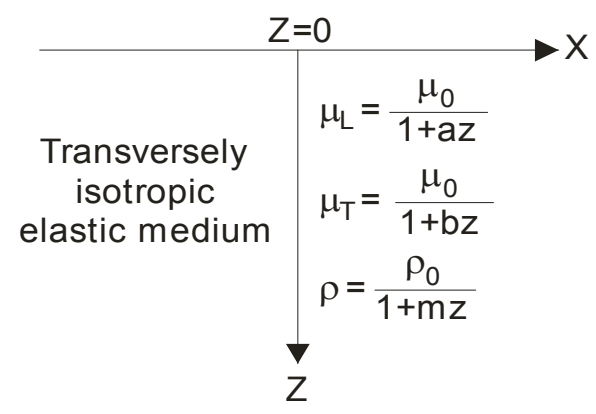

Fig.1. Geometry of the problem.

The constitutive equations for a fibre-reinforced linearly elastic anisotropic medium with respect to a preferred direction $\boldsymbol{a}$ are (Belfield et al. [12])

$$
\begin{aligned}
& \sigma_{i j}=\lambda e_{k k} \delta_{i j}+2 \mu_{T} e_{i j}+\alpha\left(a_{k} a_{m} e_{k m} \delta_{i j}+e_{k k} a_{i} a_{j}\right)+ \\
& 2\left(\mu_{L}-\mu_{T}\right)\left(a_{i} a_{k} e_{k j}+a_{j} a_{k} e_{k i}\right)+\beta\left(a_{k} a_{m} e_{k m} a_{i} a_{j}\right)
\end{aligned}
$$

where $\tau_{i j}$ are the components of stress, $e_{i j}=\frac{1}{2}\left(u_{i, j}+u_{j, i}\right)$ are components of strain, $\mu_{L}$ and $\mu_{T}$ are elastic shear moduli in longitudinal and transverse direction, $\alpha, \beta$ are reinforced anisotropic elastic parameters, $\lambda$ is the elastic parameter, $\boldsymbol{a}=\left(a_{1}, a_{2}, a_{3}\right)$ are the components of a referred to the cylindrical coordinate system.

The origin of the cylindrical coordinate system $(r, \theta, z)$ is located at the interface of the rigid layer and the $z$-axis is directly downwards. The following variation in the rigidity and the mass density are taken into account

$$
\begin{aligned}
& \mu_{L}=\frac{\mu_{0}}{1+a z}, \\
& \mu_{T}=\frac{\mu_{0}}{1+b z}, \\
& \rho=\frac{\rho_{0}}{1+m z} \quad \text { where } \quad a>0, \quad b>0, \quad m>0
\end{aligned}
$$

where $a, b$, and $m$ are constants having dimensions that are inverse of length.

The dynamic equations of motion are 


$$
\begin{aligned}
& \frac{\partial \sigma_{r r}}{\partial r}+\frac{1}{r} \frac{\partial \sigma_{r \theta}}{\partial \theta}+\frac{\partial \sigma_{r z}}{\partial z}+\frac{\sigma_{r r}-\sigma_{\theta \theta}}{r}=\rho \frac{\partial^{2} u}{\partial t^{2}}, \\
& \frac{\partial \sigma_{r \theta}}{\partial r}+\frac{1}{r} \frac{\partial \sigma_{\theta \theta}}{\partial \theta}+\frac{\partial \sigma_{\theta z}}{\partial z}+\frac{2 \sigma_{r \theta}}{r}=\rho \frac{\partial^{2} v}{\partial t^{2}}, \\
& \frac{\partial \sigma_{r z}}{\partial r}+\frac{1}{r} \frac{\partial \sigma_{\theta z}}{\partial \theta}+\frac{\partial \sigma_{z z}}{\partial z}+\frac{\sigma_{r z}}{r}=\rho \frac{\partial^{2} w}{\partial t^{2}}
\end{aligned}
$$

where $\sigma_{r r}, \sigma_{\theta \theta}, \sigma_{z z}, \sigma_{r z}, \sigma_{r \theta}$, and $\sigma_{\theta z}$, are the respective stress components, and $u, v$, and $w$ are the respective displacement components. Now, the stress-strain relations are given by

$$
\begin{aligned}
& \sigma_{r r}=\lambda \Omega+2 \mu e_{r r}, \quad \sigma_{\theta \theta}=\lambda \Omega+2 \mu e_{\theta \theta}, \\
& \sigma_{z z}=\lambda \Omega+2 \mu e_{z z}, \quad \sigma_{r \theta}=2 \mu e_{r \theta}, \\
& \sigma_{r z}=2 \mu e_{r z}, \quad \sigma_{\theta z}=2 \mu e_{\theta z}
\end{aligned}
$$

where $\lambda$ and $\mu$ are Lame's constant, and $\Omega=\left(\frac{\partial u}{\partial r}+\frac{1}{r} \frac{\partial v}{\partial \theta}+\frac{u}{r}+\frac{\partial w}{\partial z}\right)$ denotes the dilatation. The straindisplacement relations are

$$
\begin{aligned}
& e_{r r}=\frac{1}{2} \frac{\partial u}{\partial r}, \quad e_{\theta \theta}=\frac{1}{2}\left(\frac{1}{r} \frac{\partial v}{\partial \theta}+\frac{u}{r}\right), \quad e_{z z}=\frac{1}{2} \frac{\partial w}{\partial z}, \\
& e_{r \theta}=\frac{1}{2}\left(\frac{1}{r} \frac{\partial u}{\partial \theta}+\frac{\partial v}{\partial r}-\frac{v}{r}\right), \quad e_{\theta z}=\frac{1}{2}\left(\frac{1}{r} \frac{\partial v}{\partial z}+\frac{1}{r} \frac{\partial w}{\partial \theta}\right), \\
& e_{z r}=\frac{1}{2}\left(\frac{\partial w}{\partial r}+\frac{\partial u}{\partial z}\right) .
\end{aligned}
$$

The torsional wave is characterized by the displacements

$$
u=0, \quad w=0, \quad v=v(r, z, t) .
$$

Now, considering Eqs (2.4)-(2.6), the dynamical equations of motion for torsional surface waves propagating in the radial direction can be written as

$$
\frac{\partial \sigma_{r \theta}}{\partial r}+\frac{\partial \sigma_{z \theta}}{\partial z}+\frac{2 \sigma_{r \theta}}{r}=\rho(z) \frac{\partial^{2} v}{\partial t^{2}}
$$

where $v(r, z, t)$ is the displacement along the $\theta$ (azimuthal) direction, and $r$ is the radial coordinate. The stresses are related to the displacement component by 


$$
\sigma_{r \theta}=\mu(z)\left(\frac{\partial v}{\partial r}-\frac{v}{r}\right), \quad \sigma_{z \theta}=\mu(z)\left(\frac{\partial v}{\partial z}\right)
$$

Using Eq.(2.8), Eq.(2.7) takes the form

$$
\mu_{T}(z)=\left(\frac{\partial^{2}}{\partial r^{2}}+\frac{1}{r} \frac{\partial}{\partial r}-\frac{1}{r^{2}}\right) v+\frac{\partial}{\partial z}\left(\mu_{L}(z) \frac{\partial v}{\partial z}\right)=\rho(z) \frac{\partial^{2} v}{\partial t^{2}}
$$

The harmonic wave solution of Eq.(2.9) is of the form

$$
v=V(z) J_{l}(\mathrm{Kr}) \exp (i \omega t)
$$

where $\omega$ is the angular frequency, $K$ is the angular wave number, and $J_{I}(\mathrm{Kr})$ is the first order Bessel function of first kind.

Substituting Eq.(2.10) in Eq.(2.9) and using Eq.(2.2), we have

$$
\frac{d^{2} V}{d z^{2}}-\frac{a}{1+a z} \frac{d V}{d z}-K^{2}\left(1-\frac{c^{2}}{c_{0}^{2}} \frac{1+b z}{1+m z}\right) \frac{1+a z}{1+b z} V(z)=0
$$

where $c=\omega / K$ is the torsional wave velocity and $c_{0}{ }^{2}=\mu_{0} / \rho_{0}$

Now, substituting $V(z)=\frac{g(z)}{(1+a z)^{-1 / 2}}$ into Eq.(2.11) to eliminate the term $\frac{d V}{d z}$, we have

$$
\frac{d^{2} g(z)}{d z^{2}}+\left[-\frac{3 a^{2}}{4(1+a z)^{2}}-K^{2}\left(1-\frac{c^{2}}{c_{0}^{2}} \frac{1+b z}{1+m z}\right) \frac{1+a z}{1+b z}\right] g(z)=0
$$

Introducing the quantities

$$
\gamma=\sqrt{\frac{a}{b}-\frac{c^{2}}{c_{0}^{2}} \frac{a}{m}}
$$

and

$$
\eta=\frac{2 \gamma K(1+a z)}{a}
$$

in Eq.(2.12), we have

$$
\frac{d^{2} g}{d z^{2}}+\left(-\frac{1}{4}-\frac{3}{4 \eta^{2}}+\frac{R}{\eta}\right) g(\eta)=0, \quad R=\frac{K a}{2 \gamma}\left(\frac{1}{b^{2}}\left(1-\frac{b}{a}\right)-\frac{c^{2}}{c_{0}^{2}}\left(1-\frac{m}{a}\right) \frac{1}{m^{2}}\right),
$$

which is the well known Whittaker's equation (see Whittaker and Waston [27]).

The solution of Whittaker's Eq.(2.13) is given by

$$
g(\eta)=A W_{R, l}(\eta)+B W_{-R, l}(-\eta)
$$


where $W_{R, I}(\eta)$ and $W_{-R, I}(-\eta)$ are Whittaker functions.

So for the heterogeneous half -space, the solution should vanish at $z \rightarrow \infty$ i.e., $\eta \rightarrow \infty$.

So in view of the above condition the solution may be taken as

$$
g(\eta)=A W_{R, I}(\eta)
$$

Hence, the displacement for the torsional wave in the heterogeneous half-space is

$$
v=A W_{R, 1}(\eta)(1+a z)^{1 / 2} J_{1}(K r) e^{i \omega t}, \quad \eta=\frac{2 \gamma K}{a}(1+a z) .
$$

\section{Boundary condition}

At the interface $z=0$, displacement for the torsional wave vanishes, i.e.,

$$
v=0 \quad \text { at } \quad z=0 .
$$

Expanding Whittaker function up to the linear term and using the boundary condition (3.1), one finds the wave velocity equation from Eq.(2.14) as

$$
\sqrt{\frac{2 \gamma K}{a}}\left[1+(3-2 R) \frac{\gamma K}{3 a}\right] e^{-\frac{\gamma K}{a}}=0 .
$$

After simplification, we have

$$
\frac{c}{c_{0}}=\sqrt{\frac{m}{b}}
$$

or

$$
\begin{aligned}
& \frac{c_{4}}{c_{0}^{4}}\left(1-\frac{m}{a}\right)^{2}+\frac{c^{2}}{c_{0}^{2}}\left[-2\left(1-\frac{m}{a}\right)\left(1-\frac{b}{a}\right) \frac{m^{2}}{b^{2}}+\frac{6 m^{2}}{K^{2}}\left(1-\frac{m}{a}\right)+\frac{9 m^{3}}{a K^{2}}\right]+ \\
& +\frac{m^{4}}{b^{4}}\left(1-\frac{b}{a}\right)^{2}-6 \frac{m^{4}}{K^{2} b^{2}}\left(1-\frac{b}{a}\right)-\frac{9 m^{4}}{K^{2} a b}+\frac{9 m^{4}}{K^{4}}=0 .
\end{aligned}
$$

\section{Particular cases}

Case I: When $m \rightarrow 0$, i.e., medium is of constant density and all the rigidity components vary inversely linearly with depth, Eq.(3.2) reduces to, $c / c_{0} \rightarrow 0$, and torsional waves do not propagate in such a medium.

Case II: When $a=b$, i.e., both rigidities components are same, Eq.(3.2) reduces to

$$
\frac{c_{4}}{c_{0}^{4}}\left(1-\frac{m}{a}\right)^{2}+\frac{c^{2}}{c_{0}^{2}}\left[6 \frac{m^{2}}{K^{2}}\left(1-\frac{m}{a}\right)+\frac{9 m^{3}}{a K^{2}}\right]-\frac{9 m^{4}}{K^{2} a^{2}}+\frac{9 m^{4}}{K^{4}}=0 .
$$

Case III: When $a=b=m$, i.e., both rigidity components and density are same throughout the medium, then Eq.(4.1) reduces to, 


$$
\frac{c}{c_{0}}=\sqrt{1-\frac{a^{2}}{K^{2}}}
$$

\section{Numerical computations and discussion}

In order to demonstrate the effect of dispersion of non-homogeneities on the propagation of torsional surface wave in non-homogeneous transverse isotropic elastic half-space under a rigid layer, phase velocity is calculated numerically with the help of MATLAB for different particular cases from Eqs (3.2), (3.3) and (4.1). Effects of non-homogeneity parameters on torsional surface waves in a non-homogeneous transverse isotropic elastic half-space under a rigid layer are discussed in the following way by means of graphs.

Figure 2, shows the effects of non-homogeneity parameters involved in longitudinal rigidity, i.e., $K / a$, transverse rigidity, i.e., $K / b$ and density, i.e., $K / m$ of the transverse isotropic elastic half-space under a rigid layer. Here, the value of the dimensionless phase velocity $\left(c / c_{0}\right)$ has been computed from Eq.(3.2). The following observations and effects are obtained under the above considered values.

(1a) The dimensionless phase velocity $\left(c / c_{0}\right)$ of torsional surface waves increases as the value of nonhomogeneity in transverse rigidity, i.e., $K / b$, increases from 0.5 to 2.0 as shown in Fig.2.

(1b) The dimensionless phase velocity $\left(c / c_{0}\right)$ of torsional surface waves decreases as the value of nonhomogeneity in density, i.e., $K / m$, increases from 2.0 to 4.0 as shown in Fig.2.

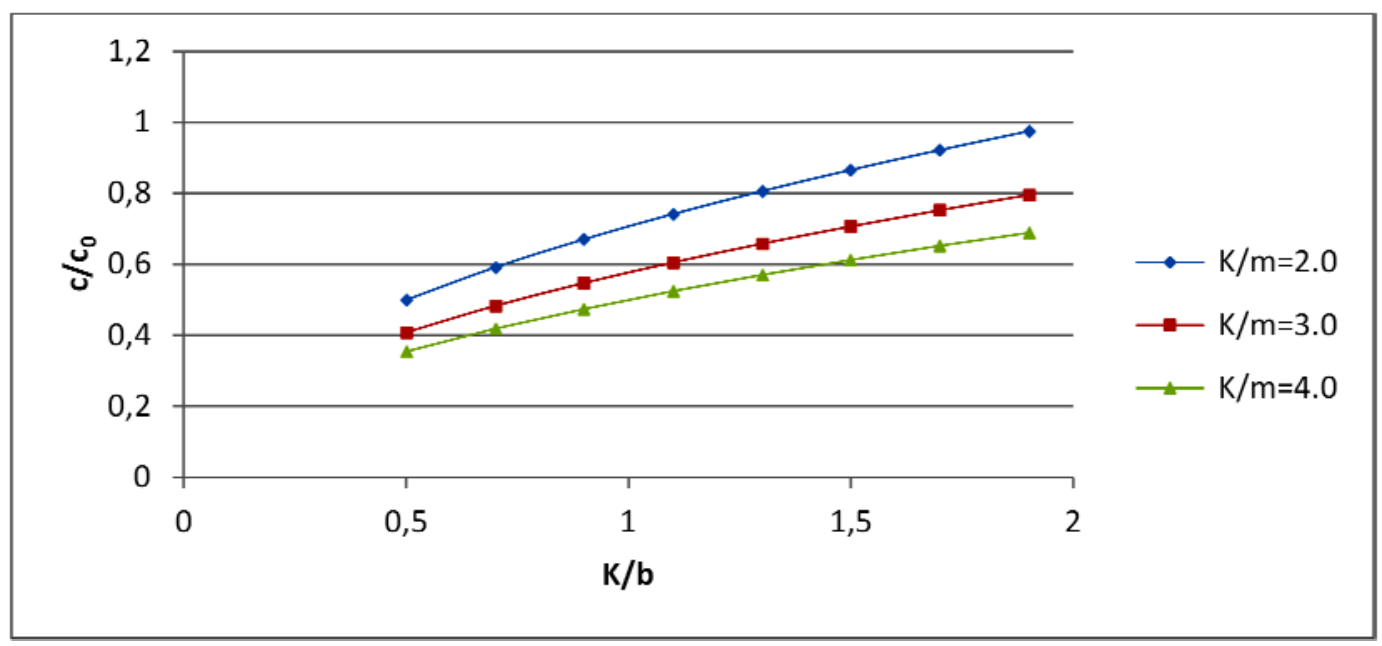

Fig.2. Variation of dimensionless phase velocity $c / c_{0}$ against dimensionless $K / b$ evaluated from Eq.(3.2) demonstrating the influence of non-homogeneity associated with density.

Figures 3-5, shows the effects of non-homogeneity parameters involved in longitudinal rigidity, i.e., $K / a$, transverse rigidity, i.e., $K / b$ and density, i.e., $K / m$, of the transverse isotropic elastic half-space under a rigid layer. Here, the value of the dimensionless phase velocity $\left(c / c_{0}\right)$ has been computed from Eq.(3.3). Following observations and effects are obtained under the above considered values.

(2a) For a fixed value of non-homogeneity in transverse rigidity i.e., $K / b=3.0$, the dimensionless phase velocity $\left(c / c_{0}\right)$ of torsional surface waves increases, as the value of non-homogeneity in longitudinal rigidity i.e., $K / a$ increases from 0.5 to 2.0 as shown in Fig.3.

(2b) For a fixed value of non-homogeneity in transverse rigidity, i.e., $K / b=2.0$, the dimensionless phase velocity $\left(c / c_{0}\right)$ of torsional surface waves decreases, as the value of non-homogeneity in density, i.e., $K / m$, increases from 3.0 to 5.0 as shown in Fig.3. 
(3a) For a fixed value of non-homogeneity in density, i.e., $K / m=3.0$, the dimensionless phase velocity $\left(c / c_{0}\right)$ of torsional surface waves increases as the value of non-homogeneity in longitudinal rigidity, i.e., $K / a$, increases from 0.5 to 2.0 as shown in Fig.4.

(3b) For a fixed value of non-homogeneity in density, i.e., $K / m=3.0$, the dimensionless phase velocity $\left(c / c_{0}\right)$ of torsional surface waves increases as the value of non-homogeneity in transverse rigidity, i.e., $K / b$, increases from 2.5 to 3.5 as shown in Fig.4.

(4a) For a fixed value of non-homogeneity in longitudinal rigidity, i.e., $K / a=1.0$, the dimensionless phase velocity $\left(c / c_{0}\right)$ of torsional surface waves increases as the value of non-homogeneity in transverse rigidity, i.e., $K / b$, increases from 2.0 to 4.0 as shown in Fig.5.

(4b) For a fixed value of non-homogeneity in longitudinal rigidit,y i.e., $K / a=2.0$, the dimensionless phase velocity $\left(c / c_{0}\right)$ of torsional surface waves decreases as the value of non-homogeneity in density, i.e., $K / m$, increases from 3.0 to 5.0 as shown in Fig.5.

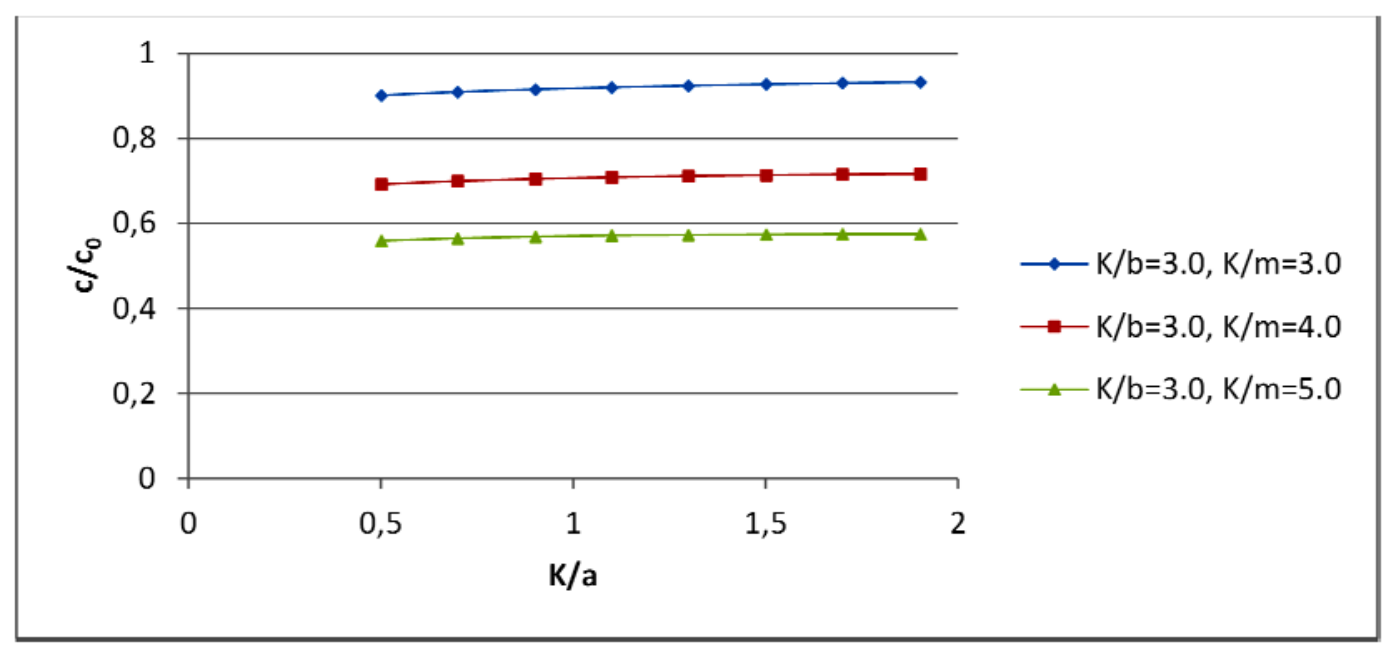

Fig.3. Variation of dimensionless phase velocity $c / c_{0}$ against dimensionless $K / a$ evaluated from Eq.(3.3) demonstrating the influence of non-homogeneity associated with density for fixed transverse rigidity.

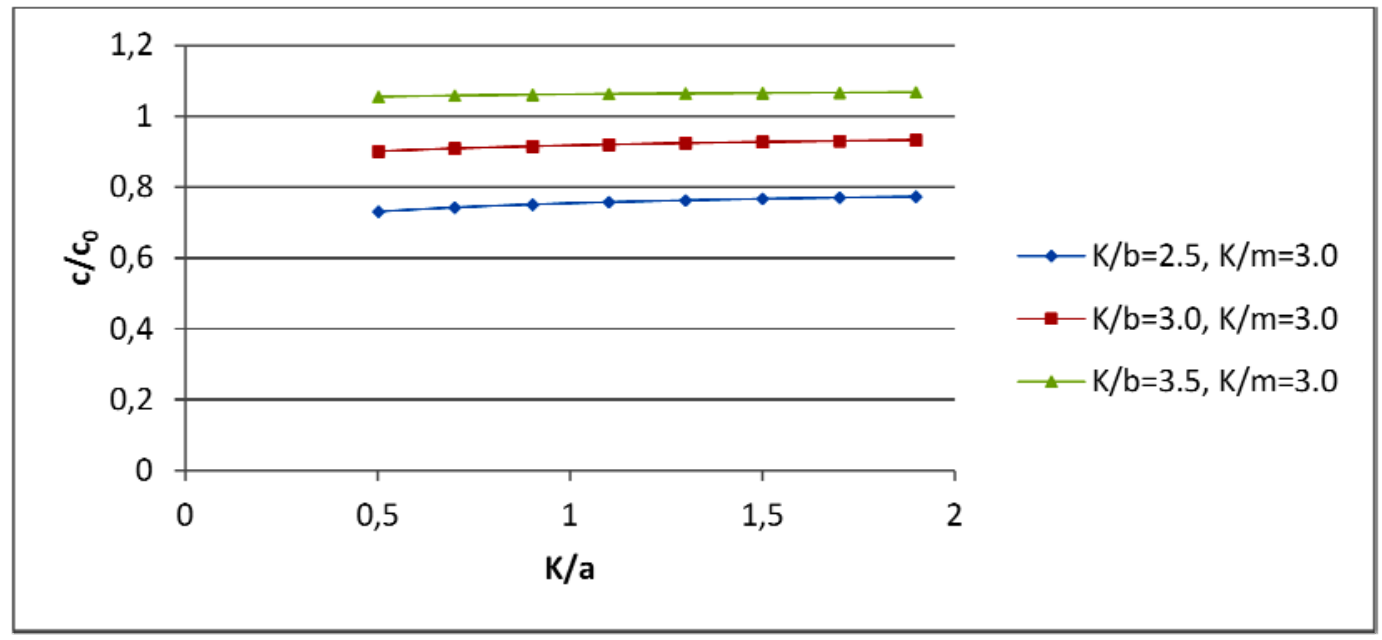

Fig.4. Variation of dimensionless phase velocity $c / c_{0}$ against dimensionless $K / a$ evaluated from Eq.(3.3) demonstrating the influence of non-homogeneity associated with transverse rigidity for fixed density. 


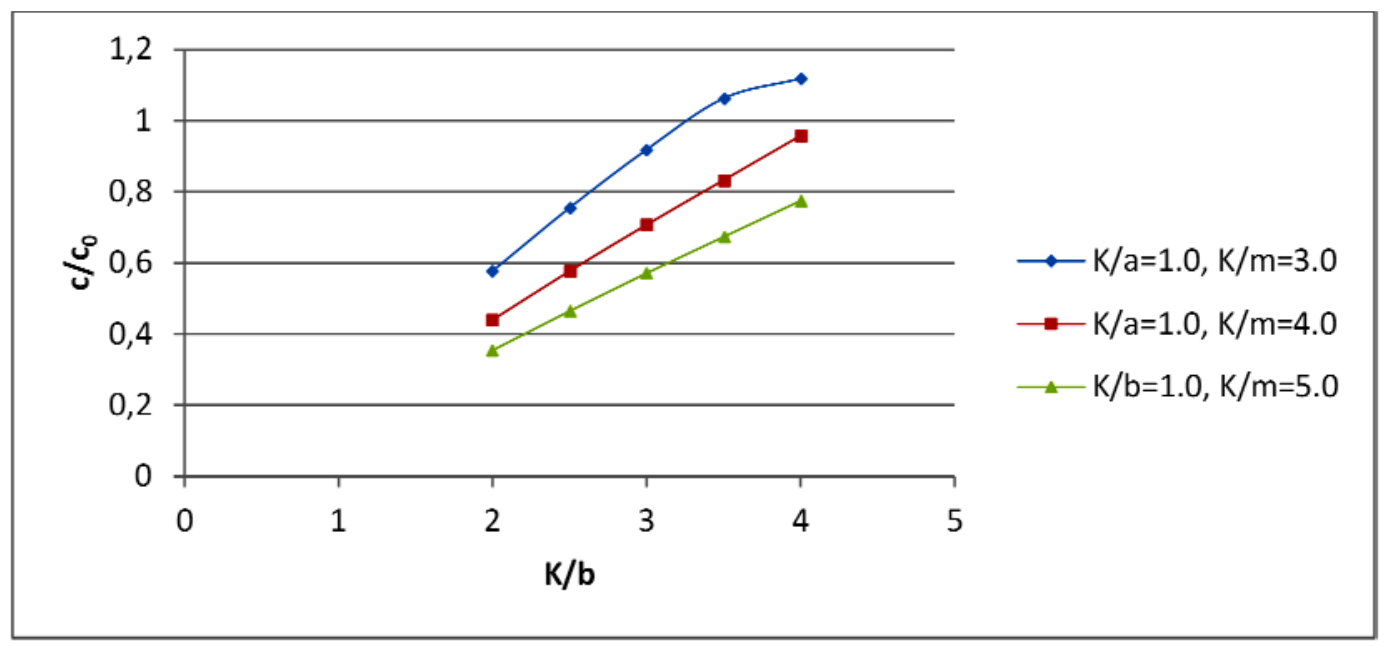

Fig.5. Variation of dimensionless phase velocity $c / c_{0}$ against dimensionless $K / b$ evaluated from Eq.(3.3) demonstrating the influence of non-homogeneity associated with density for fixed longitudinal rigidity.

Figure 6-7, show the effects of non-homogeneity parameters involved in rigidity, i.e., $K / a$ and density, i.e., $\mathrm{K} / \mathrm{m}$, of the transverse isotropic elastic half-space under a rigid layer in case of equal longitudinal and transverse rigidities. Here, the value of the dimensionless phase velocity $\left(c / c_{0}\right)$ has been computed from Eq.(4.1). The following observations and effects are obtained under the above considered values.

(5a) The dimensionless phase velocity $\left(c / c_{0}\right)$ of torsional surface waves increases as the value of nonhomogeneity in longitudinal rigidity, i.e., $K / a$, increases from 1.0 to 2.5 as shown in Fig.6.

(5b) The dimensionless phase velocity $\left(c / c_{0}\right)$ of torsional surface waves decreases as the value of non-homogeneity in density, i.e., $K / m$, increases from 3.0 to 5.0 as shown in Fig.6.

(6a) The dimensionless phase velocity $\left(c / c_{0}\right)$ of torsional surface waves decreases as the value of non-homogeneity in density, i.e., $K / m$ increases from 1.0 to 5.0 as shown in Fig.7.

(6b) The dimensionless phase velocity $\left(c / c_{0}\right)$ of torsional surface waves increases as the value of nonhomogeneity in longitudinal rigidity, i.e., $K / a$, increases from 2.0 to 3.0 as shown in Fig.7.

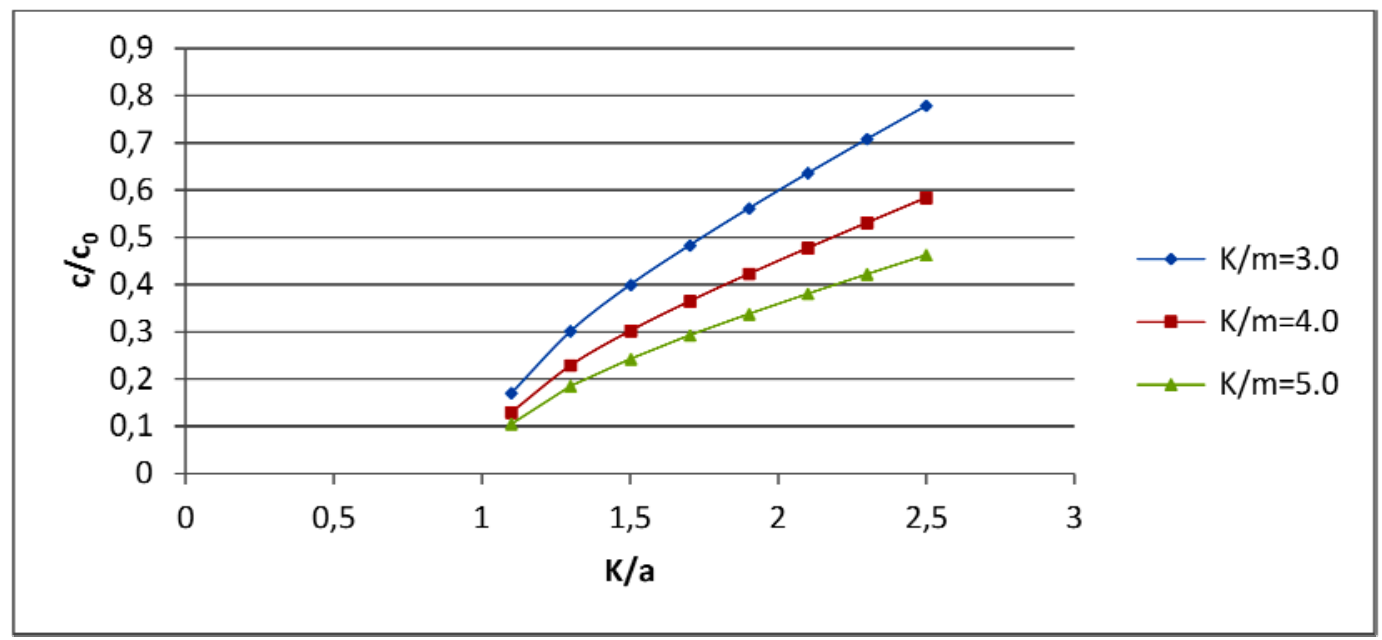

Fig.6. Variation of dimensionless phase velocity $c / c_{0}$ against dimensionless $K / a$ evaluated from Eq.(4.1) demonstrating the influence of non-homogeneity associated with density. 


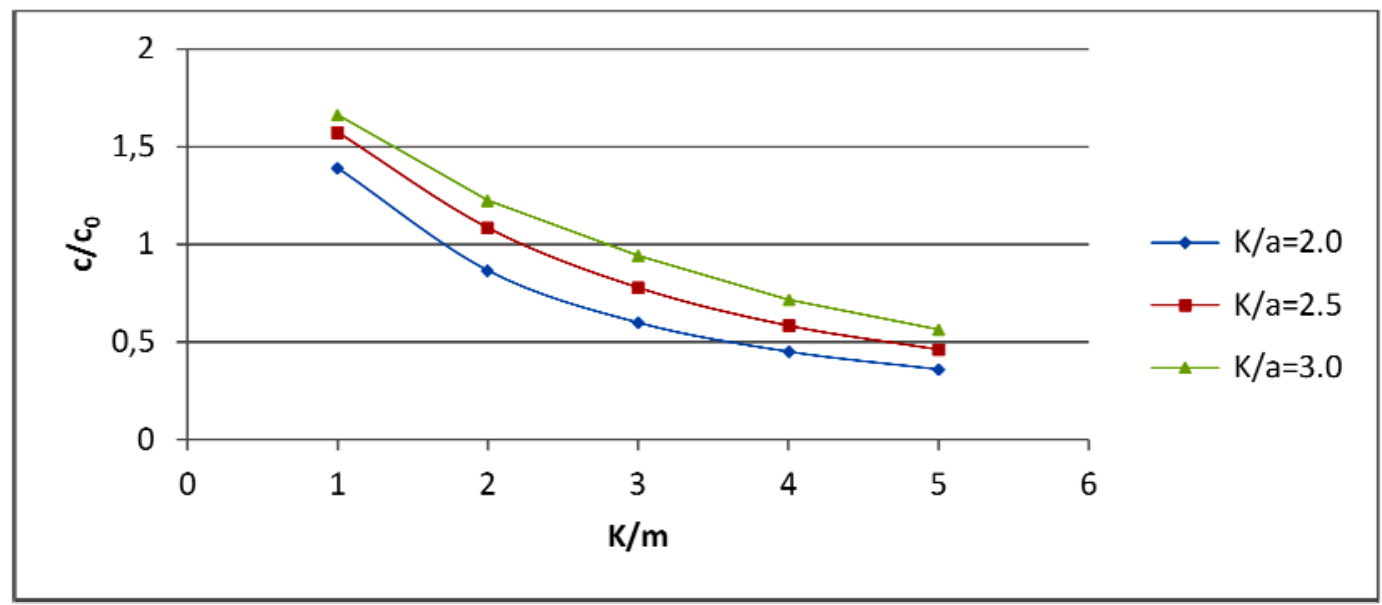

Fig.7. Variation of dimensionless phase velocity $c / c_{0}$ against dimensionless $K / m$ evaluated from Eq.(4.1) demonstrating the influence of non-homogeneity associated with longitudinal rigidity.

\section{Conclusions}

We have investigated the torsional surface wave in non-homogeneous transverse isotropic elastic half-space under a rigid layer. A closed form solution has been derived for the displacement in a half-space. By using the asymptotic expansion of Whittaker's function, we have derived the wave velocity equation for the torsional surface waves in compact form. Dimensionless phase velocity is calculated numerically for different particular cases from Eqs (3.2), (3.3), and (3.4) with the help of MATLAB. The effects of various dimensionless elastic parameters and non-homogeneity factors on the dimensionless phase velocity $\left(c / c_{0}\right)$ have been shown graphically. We make the following conclusions

1. There are two torsional wave-fronts propagating in a transversely isotropic elastic half-space with exponentially varying rigidity and density under a rigid layer, one of which is shown in Figs 2 and the other is shown in Figs 3-5.

2. The dimensionless phase velocity $\left(c / c_{0}\right)$ of torsional surface waves increases as the value of nonhomogeneity in transverse rigidity increases.

3. The dimensionless phase velocity $\left(c / c_{0}\right)$ of torsional surface waves decreases as the value of nonhomogeneity in density increases.

4. For a fixed transverse rigidity, the dimensionless phase velocity $\left(c / c_{0}\right)$ of torsional surface waves increases as the value of non-homogeneity in longitudinal rigidity increases.

5. For a fixed transverse rigidity, the dimensionless phase velocity $\left(c / c_{0}\right)$ of torsional surface waves decreases as the value of non-homogeneity in density increases.

6. For a fixed density, the dimensionless phase velocity $\left(c / c_{0}\right)$ of torsional surface waves increases as the value of non-homogeneity in longitudinal rigidity increases.

7. For a fixed density, the dimensionless phase velocity $\left(c / c_{0}\right)$ of torsional surface waves increases as the value of non-homogeneity in transverse rigidity increases.

8. In the case of same longitudinal and transverse rigidities, the dimensionless phase velocity $\left(c / c_{0}\right)$ of torsional surface waves increases as the longitudinal rigidity increases and density decreases.

9. In the case of a medium of constant density and all the rigidity components vary inversely linearly with depth, the torsional surface waves do not propagate in such a medium.

This study may be useful in understanding the behavior of seismic waves generated by artificial explosions and helps to find the cause and estimate of damage due to earthquake. The present paper may be useful in predicting the behavior of torsional surface waves in transverse elastic media. 


\section{Nomenclature}

$$
\begin{aligned}
a, b, m & - \text { non-homogeneity parameters in medium } \\
c & - \text { velocity of torsional waves } \\
e_{\mathrm{rr}}, e_{\theta \theta}, e_{\mathrm{zz}}, & - \text { strain components in cylindrical coordinates } \\
e_{\mathrm{rz}}, e_{r \theta}, e_{\theta z} & \\
K & \text { - wave number } \\
v(r, z, t) & - \text { displacement along the azimuthal direction } \\
\lambda, \mu & - \text { Lame's constants } \\
\mu_{L} & - \text { elastic shear modules in longitudinal direction } \\
\mu_{T} & - \text { elastic shear modules in transverse direction } \\
\sigma_{\mathrm{rr}}, \sigma_{\theta \theta}, \sigma_{\mathrm{zz}}, & - \text { stress components in cylindrical coordinates } \\
\sigma_{\mathrm{rz}}, \sigma_{\mathrm{r} \theta}, \sigma_{\theta \mathrm{z}} & \text { - density of semi-infinite medium } \\
\Omega & \text { - dilatation }
\end{aligned}
$$

\section{References}

[1] Stoneley R. (1924): Elastic waves at surface of separation of two solids. - Proc. R. Soc. A 806. pp.416-428.

[2] Bullen K.E. (1965): Theory of Seismology. - Cambridge University Press.

[3] Ewing W.M., Jardetzky W.S. and Press F. (1957): Elastic Waves in Layered Media. - New York: Mcgraw-Hill.

[4] Hunter S.C. (1970): Viscoelastic Waves, Progress in Solid Mechanics, I. - (ed: Sneddon IN and Hill R) Cambridge University Press.

[5] Jeffreys H. (1970): The Earth. - Cambridge University Press.

[6] Sezawa K. (1927): Dispersion of elastic waves propagated on the surface of stratified bodies and on curved surfaces. - Bull. Earthq. Res. Inst. Tokyo, vol.3. pp.1-18.

[7] Thomson W.J. (1950): Transmission of elastic waves through a stratified elastic medium. - Appl. Phys., vol.21, pp.89-93.

[8] Haskell N.A. (1953): The dispersion of surface waves in multilayered media. - Bull. Seis. Soc. Amer., vol.43. pp.17-34.

[9] Biot M.A. (1965): Mechanics of Incremental Deformations. J. Willy.

[10] Sinha N. (1967): Propagation of Love waves in a non-homogeneous stratum of finite depth sandwiched between two semi-infinite isotropic media. - Pure Applied Geophysics, vol.67, pp.65-70.

[11] Roy P.P. (1984): Wave propagation in a thin two layered medium with stress couples under initial stresses. - Acta Mechanics, vol.54. pp.1-21.

[12] Belfield A.J., Rogers T.G. and Spencer A.J.M. (1983): Stress in elastic plates reinforced by fibers lying in concentric circles. - Journal of the Mechanics and Physics of Solids, vol.31, No.1, pp.25-54.

[13] Datta B.K. (1986): Some observation on interactions of Rayleigh waves in an elastic solid medium with the gravity field. - Rev. Roumaine Sci. Tech. Ser. Mec. Appl., vol.31. pp.369-374.

[14]Chattopadhyay A., Chakraborty M. and Pal A.K. (1983): Effects of irregularity on the propagation of guided SH waves. - Jr. de Mecanique Theo. et appl, Vol.2 No.2. pp.215-225.

[15] Goda M.A. (1992): The effect of inhomogeneity and anisotropy on Stoneley waves. - Acta Mech., vol.93, No.1-4. pp.89-98.

[16] Gupta S., Vishwakarma S.K., Majhi D.K. and Kundu S. (2012): Influence of linearly varying density and rigidity on torsional waves in inhomogeneous crustal layer. - Appl. Math. Mech.-Engl. Ed., vol.33, No.10. pp.1239-1252. 
[17] Georgiadis H.G., Vardaulakis I. and lykotrafitis G. (2000): Torsional surface wave in gradient-elastic half-space. Wave Motion, vol.31, No.4, pp.333-348.

[18] Dey S. and Sarkar M.G. (2002): Torsional surface waves in an initially stressed anisotropic porous medium. - J. Eng. Mech., vol.128, No.2, pp.184-189.

[19] Selim M.M. (2007): Propagation of torsional surface wave in heterogeneous half-space with irregular free surface. - Appl. Math. Sci., vol.1, No.29-32, pp.1429-1437.

[20] Ozturk A. and Akbbarov S.D. (2009): Torsional wave propagation in a pre-stressed circular cylinder embedded in a pre-stressed elastic medium. - Appl. Math. Model., vol.33. pp.3636-3649.

[21] Gupta S., Majhi D.K., Kundu S. and Vishwakarma S.K. (2012): Propagation of torsional surface waves in a homogeneous layer of finite thickness over an initially stressed heterogeneous half-space. - Appl. Math. Comput., vol.218. pp.5655-5664.

[22] Davini C., Paroni R. and Puntle E. (2008): An asymptotic approach to the torsional problem in thin rectangular domains. - Meccanica, vol.43, No.4, pp.429-435.

[23] Dey S., Gupta S., Gupta A.K., Kar S.K. and De P.K. (2003): Propagation of torsional surface waves in an elastic layer with void pores over an elastic half-space with void pores. - Tamkang J. Sci. Eng., vol.6, No.4, pp.241-249.

[24] Sethi M., Gupta K.C., Rani Monika and Vasudeva A. (2013): Surface waves in homogeneous viscoelastic media of higher order under the influence of surface stresses. - J. Mech. Behav. Mater., vol.22, No.5-6, pp.185-191.

[25] Sethi M., Sharma Arvind and Sharma Anupamdeep (2016): Propagation of SH waves in a double homogeneous crustal layers of finite depth lying over an homogeneous half-space. - Latin American journal of Solids and Structures, vol.13, No.14, pp.2328-2342.

[26] Sethi M. and Sharma Anupamdeep (2016): Propagation of SH waves in an regular non homogeneous monoclinic crustal layer lying over a non-homogeneous semi-infinite medium. - International Journal of Applied Mechanics and Engineering, vol.21, No.1, pp.447-459.

[25] Whittaker E.T. and Watson G.N. (1990): A Course in Modern Analysis. - $4^{\text {th }}$ Edn, Cambridge: Cambridge University Press.

Received: April 6, 2016

Revised: December 16, 2016 